$1-1-2013$

\title{
Insight into morphology changes of nanoparticle laden droplets in acoustic field
}

Saptarshi Basu

Erick Tijerino

Ranganathan Kumar

University of Central Florida

Find similar works at: https://stars.library.ucf.edu/facultybib2010 University of Central Florida Libraries http://library.ucf.edu

This Article is brought to you for free and open access by the Faculty Bibliography at STARS. It has been accepted for inclusion in Faculty Bibliography 2010 s by an authorized administrator of STARS. For more information, please contact STARS@ucf.edu.

\section{Recommended Citation}

Basu, Saptarshi; Tijerino, Erick; and Kumar, Ranganathan, "Insight into morphology changes of nanoparticle laden droplets in acoustic field" (2013). Faculty Bibliography 2010 s. 3671.

https://stars.library.ucf.edu/facultybib2010/3671

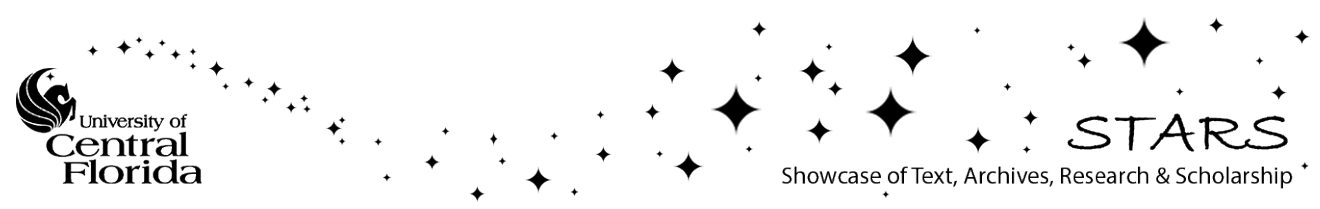




\section{Insight into morphology changes of nanoparticle laden droplets in acoustic field}

Cite as: Appl. Phys. Lett. 102, 141602 (2013); https://doi.org/10.1063/1.4801502

Submitted: 14 February 2013 . Accepted: 28 March 2013 . Published Online: 09 April 2013

Saptarshi Basu, Erick Tijerino, and Ranganathan Kumar
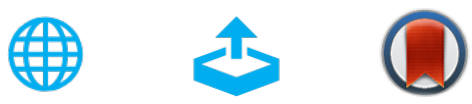

View Online

Export Citation

\section{ARTICLES YOU MAY BE INTERESTED IN}

Thermally induced secondary atomization of droplet in an acoustic field Applied Physics Letters 100, 054101 (2012); https://doi.org/10.1063/1.3680257

Acoustic levitation of soap bubbles in air: Beyond the half-wavelength limit of sound Applied Physics Letters 110, 121602 (2017); https://doi.org/10.1063/1.4979087

Sectorial oscillation of acoustically levitated nanoparticle-coated droplet Applied Physics Letters 108, 031603 (2016); https://doi.org/10.1063/1.4940143

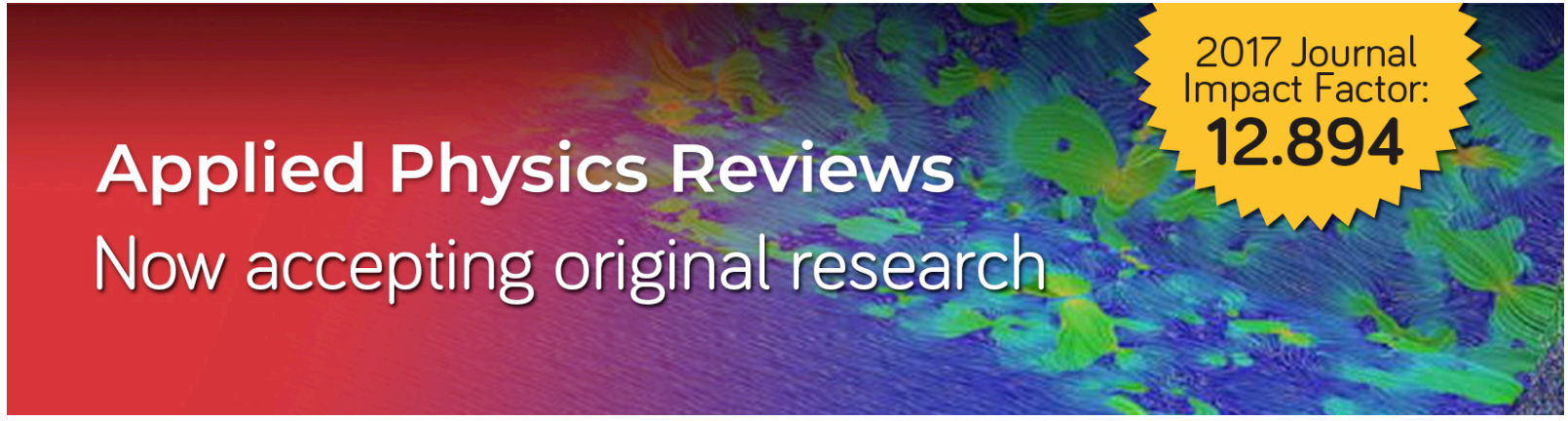




\title{
Insight into morphology changes of nanoparticle laden droplets in acoustic field
}

\author{
Saptarshi Basu, ${ }^{1, a)}$ Erick Tijerino, ${ }^{2, a)}$ and Ranganathan Kumar $^{2, a), b)}$ \\ ${ }^{1}$ Indian Institute of Science, Bangalore, India \\ ${ }^{2}$ University of Central Florida, Orlando, Florida 32816, USA
}

(Received 14 February 2013; accepted 28 March 2013; published online 9 April 2013)

\begin{abstract}
Hollow structures with unique morphologies form due to particle agglomeration in acoustically levitated nanofluid functional droplets when subjected to external heating. The final diameter of the structure depends only on the ratio of agglomeration to evaporation time scales for various nanoparticle laden droplets, and not on the type of the suspended particles. These time scales depend only on nanoparticle concentration. This valuable information may be exploited to form microstructures with desired properties from ceramic compounds. Phase diagrams for alumina and silica droplets indicate the transition from a bowl to ring structure depending on concentration. (C) 2013 AIP Publishing LLC. [http://dx.doi.org/10.1063/1.4801502]
\end{abstract}

Manufacturing and engineering particles at the nano and micro scales with diversified shapes has become increasingly important for numerous special applications, such as in drug delivery, catalysts, electronics, sensors, and photonic materials. These shapes offer different and often enhanced biological, chemical, and physical properties from those of spherical or dense materials. ${ }^{1}$ Different morphologies, such as porous particles in the submicron range, have catalytic properties similar to those of nanoparticles. ${ }^{2}$ Preferential agglomeration of particles into ring-like and non ring-like patterns has been observed during evaporation of droplets containing colloidal suspension. ${ }^{3}$ In biological applications, spreading and evaporation DNA drops can lead to the formation of periodic structures primarily due to radial stresses. ${ }^{4}$ However, most of these methods and analyses are restricted to droplet in contact with a substrate or droplets undergoing slow drying. While these allow controlled spreading, accumulation, and agglomeration, they do not allow for rapid processing and a wide spectrum of control parameters to enable precise production of desired morphologies. In this Letter, we show that different morphologies such as bowl and ring-shaped structures can be obtained by simply manipulating the initial particle concentration, particle size, external heating rate, and acoustic levitation sound pressure level (SPL). These types of unique manipulation of structure size and geometry may be used in several applications such as in pharmaceutical and ceramic industries.

The use of container-free environment (to avoid contamination) along with the use of controlled acoustics has not been investigated in detail to generate unique morphologies and microstructures. For a stable levitation, the acoustic pressure force around the droplet should balance the weight and surface tension forces of the droplet. In a laboratory environment, the droplet shifts downwards to a location called the anti-node in the levitator. Combining the ideas of

\footnotetext{
${ }^{a}$ Saptarshi Basu, Erick Tijerino, and Ranganathan Kumar equally contributed to this work.

b) Author to whom correspondence should be addressed. Electronic mail: Ranganathan.Kumar@ucf.edu
}

acoustic levitation, ${ }^{5,6}$ nanoparticle suspension, and internal fluid dynamics at the mini scale, we provide a pathway to manufacture microstructures of controlled and desired morphology. The presence of nanoparticles agglomerating on the vaporizing droplet surface lends itself to making fine, uniform, dense, and defect-free microstructures of ceramic particles.

Based on the extensive study of droplets in acoustic field and pool boiling, ${ }^{7-11}$ our hypotheses are that (1) preferential migration, accumulation, and agglomeration of suspended nanoparticles in 300-1000 $\mu \mathrm{m}$ droplets heated by controlled laser flux will modulate the vaporization process and eventually form unconventional yet repetitive structures and (2) combinations of sound pressure (acoustic streaming), rapid heating rates, and initial particle concentration alter the physico-chemical properties, transport mechanisms of the nanoparticles, and subsequent agglomeration in the functional droplets leading to varied but controlled morphologies.

To date, literature concerning micro particle drying is empirical primarily due to the instabilities of such droplets with colloidal nanosuspensions. While initially these suspensions dry isotropically, they buckle eventually like elastic shells, leaving hollow particles with uniform and nonuniform porosity. ${ }^{12}$ Multiple shapes occur and are hard to predict or control, because the drying process of these droplets proceeds through non-equilibrium states where material properties are frequently unknown and experimentally difficult to assess. ${ }^{13}$ In addition, the large number of physical parameters, such as droplet size, viscosity of droplet, and drying temperature, makes it extremely difficult to determine the morphology of particles a priori.

Nanofluid (water as solvent) droplets of $\sim 500 \mu \mathrm{m}$ $( \pm 20 \mu \mathrm{m})$ in diameter were suspended using an acoustic levitator (Tec5 ultrasonic levitator, $100 \mathrm{kHz}$ ) at sound pressure levels ranging from $160 \mathrm{~dB}$ to $165 \mathrm{~dB}$. Droplets of different particle concentrations " $\varphi$ ", ranging from 0.5 to $5 \%$ vol., were studied [diluted from a maximum concentration: $10 \mathrm{~nm}$ silica $\varphi=7.33 \%$ vol.; $20 \mathrm{~nm}$ Silica $\varphi=23.11 \%$ vol.; $20 \mathrm{~nm}$ alumina: $\varphi=8.99 \%$ vol.; and $50 \mathrm{~nm}$ alumina $\varphi=5.99 \%$ vol.]. Droplets were levitated at a sound pressure level of 
$162 \mathrm{~dB}$. In addition, $1 \%$ vol. glycerin was added to $0.5 \%$ $10 \mathrm{~nm}$ silica to match the viscosity of $50 \mathrm{~nm} 0.5 \%$ vol. alumina suspension. Likewise, $4 \%$ glycerin was added to $2 \%$ $10 \mathrm{~nm}$ silica to match the viscosity of $50 \mathrm{~nm} 2 \%$ vol. alumina suspension. Similarly, surfactant was added to $10 \mathrm{~nm}$ and $20 \mathrm{~nm}$ silica nanofluid with concentration $\varphi=0.5 \%$ vol. to determine the effect of surface tension reduction on the final structure morphology. Such changes in properties alter the orthokinetic or shear mechanism, which can control particle transport and hence the final structure. This work is aimed at controlling the precipitation outcome of various functional droplets and unifying all the data for different nanosuspensions (with various particle sizes and chemical characteristics) using a general framework extended from our previous works. $^{7-11}$

The droplets were heated using a carbon dioxide $\left(\mathrm{CO}_{2}\right)$ laser (wavelength $=10.6 \mu \mathrm{m}$, nominal beam diameter $=2 \mathrm{~mm}$, and laser power $=0.75 \mathrm{MW} / \mathrm{m}^{2}$ ). The droplet surface temperature, ranging from $20^{\circ} \mathrm{C}$ to $70^{\circ} \mathrm{C}$, was measured using an infrared (IR) camera at $100 \mathrm{fps}$ (FLIR G3-F/2) using a $40 \mathrm{~mm}$ microscopic zoom lens $(3 \times$ magnification) at a $90^{\circ}$ angle with respect to the $\mathrm{CO}_{2}$ laser. A negligible error of $0.03{ }^{\circ} \mathrm{C}$ in the temperature measurements was found due to the change in droplet emissivity. The droplet diameter reduction and morphological changes during the experiment were captured using a high speed camera at $500 \mathrm{fps}$ (Fastec TSHRMM, with a maximum speed of 16000 frames per second) equipped with a microscopic zoom lens (Navitar 6000) at a $30^{\circ}$ angle with respect to the radiative heat source. An illustration of the experimental setup is provided in our earlier paper. ${ }^{7}$ The morphology of the precipitated samples was recorded in a scanning electron microscope (SEM).

In general, the viscosity $\mu_{l}$ increases and surface tension decreases with particle concentration (Figure 1). In the special case of the $20 \mathrm{~nm}$ alumina droplets, the dispersing agent in the suspension used to maintain stable colloidal suspension causes a sharp increase in viscosity and sharp decrease in surface tension with particle concentration. The approximate instantaneous viscosity during the experiment can be estimated by calculating the instantaneous particle concentration in the droplet. Since the particle volume within the droplet is known, the instantaneous particle concentration can be calculated using the high speed imaging data indicating the instantaneous droplet volume. Usually, the increase in viscosity is not uniform across the droplet, as the liquid evaporation occurs from the droplet surface. This localized increase in viscosity near the droplet surface affects the velocity gradient and decreases the strength of recirculation within the droplet. ${ }^{7,9}$

Orthokinetic agglomeration process which is a function of shear stress, liquid viscosity, and the internal flow structures leads to the formation of structures in nanofluid droplets. $^{9-11}$ Fluid viscosity increases with particle concentration. Three timescales can be used to characterize the structure formation results, namely evaporation, agglomeration, and deformation time scales, which were discussed in detail in our earlier work ${ }^{7}$ and reiterated here for completeness. The evaporation time scale $t_{e}$ is defined as the total time needed for complete liquid evaporation: $t_{e}=t_{f}+\frac{V_{o}-V_{f}}{\dot{V}_{f}}$, where $t_{f}$ is the time

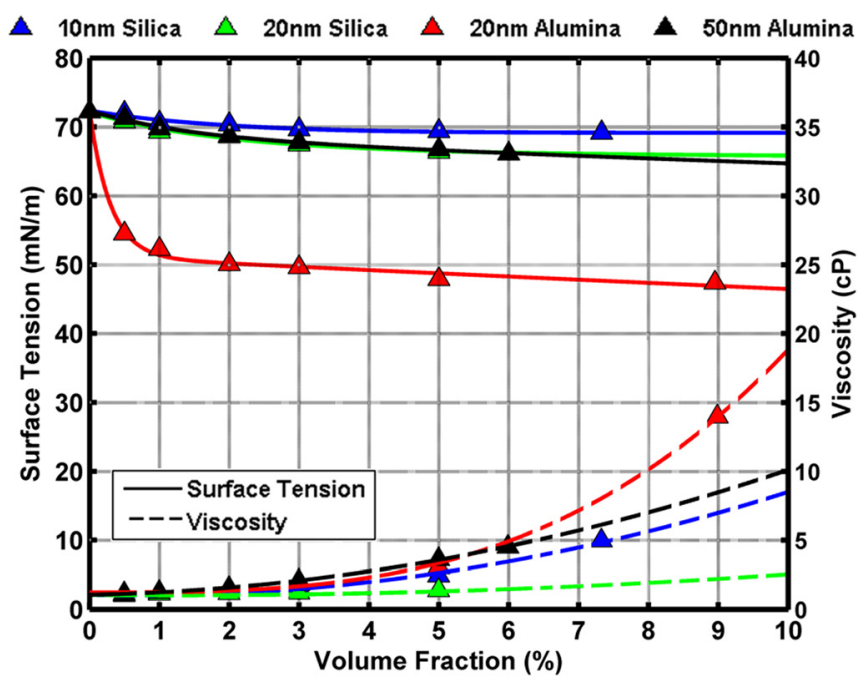

FIG. 1. (a) Viscosity $\mu$ vs. Concentration; (b) Surface Tension $\sigma$ vs. Concentration for $10 \mathrm{~nm}, 20 \mathrm{~nm}$ silica and $20 \mathrm{~nm}, 50 \mathrm{~nm}$ alumina suspensions.

at which the regression of the droplet external surface ceases with formation of outer porous crust, and $V_{o}$ and $V_{f}$ are the droplet initial and final volume respectively. The agglomeration time scale $t_{g}$ is defined as the time when particles agglomerate into a gel by means of orthokinetic agglomeration, $\mathrm{t}_{\mathrm{g}}=\frac{\pi \mathrm{D}}{4 \dot{\gamma}(3-\mathrm{D})}\left(\varphi_{\mathrm{o}}^{-1}-1\right)$, where $\varphi_{o}$ is a function of particle concentration, fractal dimensionality (D), and shear stress within the droplet $(\dot{\gamma}) \cdot{ }^{14}$ Shear stress is a function of the recirculation velocity scale $u_{l}$, the ratio of the gaseous and liquid phase viscosities, and densities denoted by $\mu_{o}, \mu_{l}$ and $\rho_{o}, \rho_{l}$, $\dot{\gamma} \sim \frac{u_{l}}{r_{d}} ; u_{l}=\sqrt{\frac{\mu_{o} \rho_{0}}{\mu_{l} \rho_{l}}} u_{\max }$. The recirculation velocity scale is a function of the acoustic Mach number: ${ }^{15} \quad S P L=197$ $+20 \log (M a)$ and the speed of sound in the gaseous phase, $u_{\max }=M a c_{o}$. The last timescale is the deformation time scale $t_{d e f}$, which is defined as the time needed for a fluid parcel $\left(m_{i}\right)$ to deform under the Bernoulli pressure through a length scale $y$ in the absence of surface tension and viscosity: $t_{\text {def }} \equiv\left[\frac{y * m_{i}}{p_{B} A_{d}}\right]^{1 / 2}$, where $\mathrm{p}_{\mathrm{B}} \mathrm{A}_{\mathrm{d}}$ is the force acting on the droplet due to acoustic pressure. The timescales developed ${ }^{7}$ can be readily extended in the current work to provide a complete description of different nanosuspensions irrespective of particle size, thermo-physical properties (like viscosity and surface tension) and type (silica, alumina, etc.).

The ratio of the evaporation and agglomeration timescales $t_{g} / t_{e}$ can describe a universal behavior of the final morphology irrespective of the initial volume concentration (Figure 2). This correlation captures the final lengthscale of the structure formed regardless of the type of nanofluids used and the differential particle sizes. The recirculation velocity scales with viscosity as $u_{l} \sim 1 / \sqrt{\mu_{l}}$. The agglomeration time scales with $u_{l}$ as $t_{g} \sim \frac{1}{u_{l}}$. This implies that $t_{g}$ scales with recirculation velocity scale as $t_{g} \sim \sqrt{\mu_{l}}$.

The reduction of recirculation within the droplet due to viscosity also increases the evaporation time scale $t_{e}$ due to the decrease of convection within the droplet, which hinders the heat transfer from the surface to the center of the droplet. The increase in evaporation time scale manifests as a longer time for structure formation $t_{f}$ (at which both $D / D_{o}$ and 


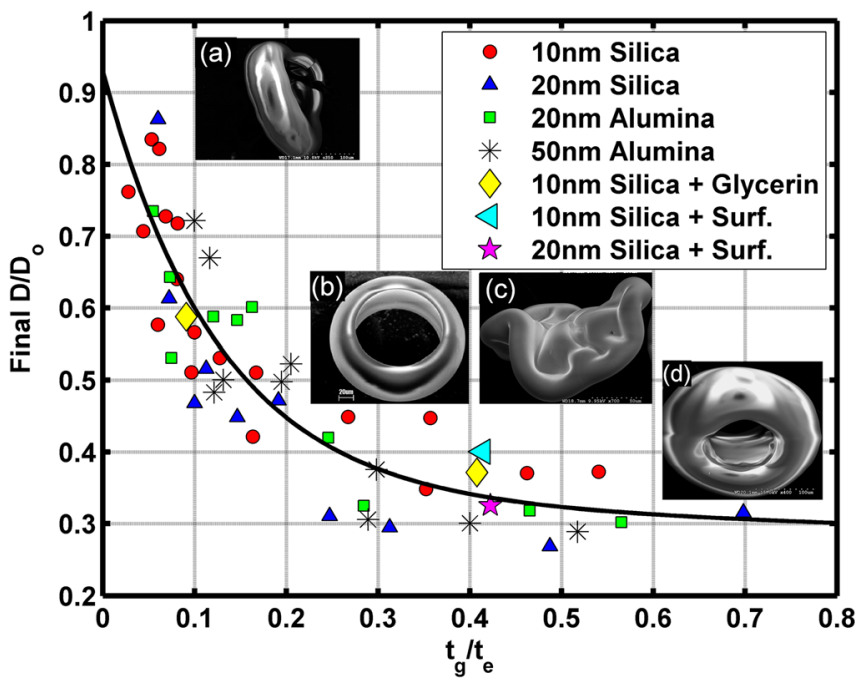

FIG. 2. Final $D / D_{o}$ vs. $t_{g} / t_{e}$ for different particle concentrations. Inset: SEM images of (a) $10 \mathrm{~nm}$ silica, (b) $20 \mathrm{~nm}$ silica, (c) $20 \mathrm{~nm}$ alumina, (d) $50 \mathrm{~nm}$ alumina. $\varphi=2 \%$ vol.

aspect ratio stabilize), which can be seen in Figure 2 for 10 $\mathrm{nm}$ silica suspensions with and without glycerin.

The surface tension of $10 \mathrm{~nm}$ silica and $20 \mathrm{~nm}$ silica at $\varphi=0.5 \%$ vol. was reduced to about $54 \mathrm{mN} / \mathrm{m}$ by adding "polisorbate 80 " surfactant at a $1 \%$ vol. concentration. This leads to an increase in aspect ratio which reduces the effective area exposed to laser heating. Surface tension reduction results in a decrease in $t_{g} / t_{e}$, similar to the enhanced viscosity cases (Figure 2). The final aspect ratio of the structure (Figure 3) is also affected by the increase in evaporation time caused by increased viscosity and reduced surface tension. When viscosity increases, the evaporation time is longer compared to the deformation time scale, yielding slightly larger aspect ratio structures. Since the laser heat flux is constant, the decrease in the projected area of the droplet causes a reduction in the total amount of heat absorbed by the droplet. This increases the evaporation timescale $\left(t_{e}\right)$. The increase of $t_{e}$ with viscosity is higher than the increase of $t_{g}$. Consequently, the increase in viscosity has an overall effect of decreasing the ratio of agglomeration and evaporation

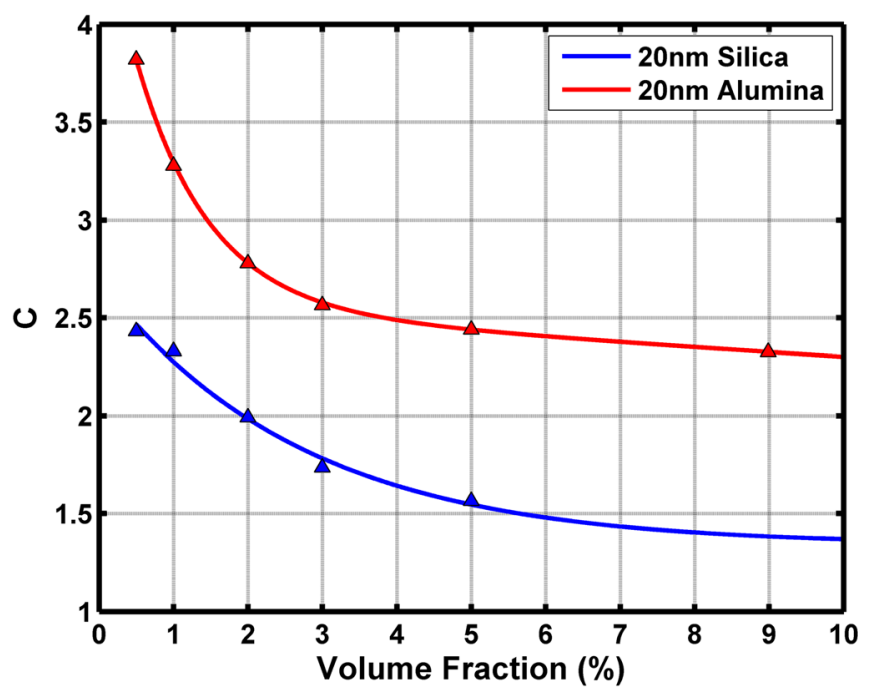

FIG. 3. Final aspect ratio, C vs. $\varphi, 20 \mathrm{~nm}$ silica and $20 \mathrm{~nm}$ alumina droplets.

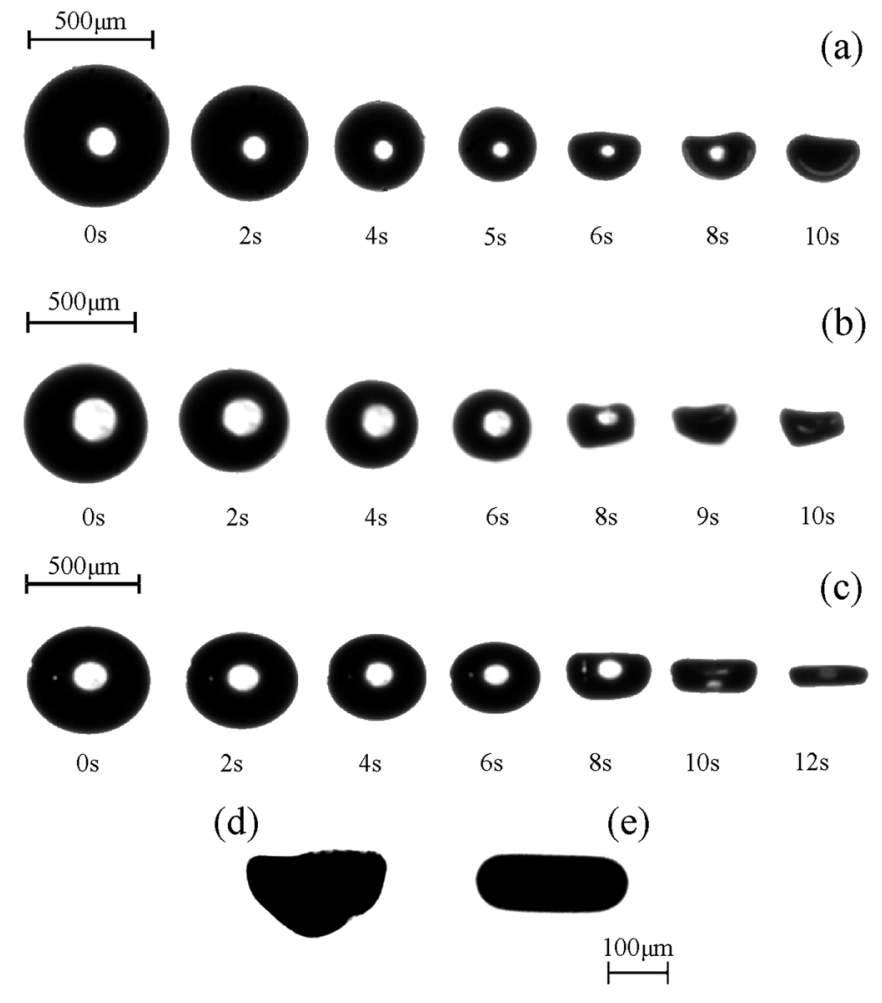

FIG. 4. Final morphologies of the precipitates for various nanofluids of different particle sizes with and without additives (surfactant and glycerin). (a) $10 \mathrm{~nm}$ silica (b) $10 \mathrm{~nm}$ silica + glycerin, (c) $50 \mathrm{~nm}$ alumina $\varphi=0.5 \%$ vol., (d) $10 \mathrm{~nm}$ silica, $\varphi=0.5 \%$ vol.; (e) $10 \mathrm{~nm}$ silica + surfactant, $\varphi=0.5 \%$ vol.

time scales $\left(t_{g} / t_{e}\right)$. Due to the relatively longer evaporation time scale compared to the agglomeration time scale, particles agglomerate faster. This yields a larger final structure $D / D_{o}$ (Figure 2). Figure 2 inset shows the SEM images of the different morphologies of silica and alumina droplets. Figure 3 shows the aspect ratio of the final precipitate for alumina and silica droplets at different initial concentrations. It is nominally seen that with increase in particle concentration, the aspect ratio shows a decrease for both alumina and silica droplets. Comparing $10 \mathrm{~nm}(\varphi=0.5 \%$ vol. $)$ silica with and without glycerin, a bowl structure (Figure 4(a)) forms for silica droplet in contrast to a ring for silica + glycerin (Figure 4(b)). The ring structure is qualitatively similar to the ring (Figure 4(d)) formed from the $50 \mathrm{~nm}$ alumina droplet (which is equal in viscosity to silica + glycerin), confirming that the viscosity plays a more pivotal role in determining the final structure even for widely different nanofluids.

The deformation time scale is reduced due to the decrease in surface free energy caused by the reduction of surface tension. This yields larger aspect ratio structures for droplets with lower surface tension. In addition to the reduction of $t_{d e f}$, the evaporation time increases due to the reduction of projected area to heating, as explained before. This leads to a further disparity in the deformation and evaporation time scales contributing to the formation of even high aspect ratio structures (Figures 3 and 4) such as rings. Figures 4(d) and 4(e) present a comparison between $10 \mathrm{~nm}$ silica at $\varphi=0.5 \%$ vol. with and without added surfactant. Bowls are formed for higher surface tension liquid drops, in contrast to the rings formed for the reduced surface tension cases (Figures 4(d) and 4(e)). A different morphology was 


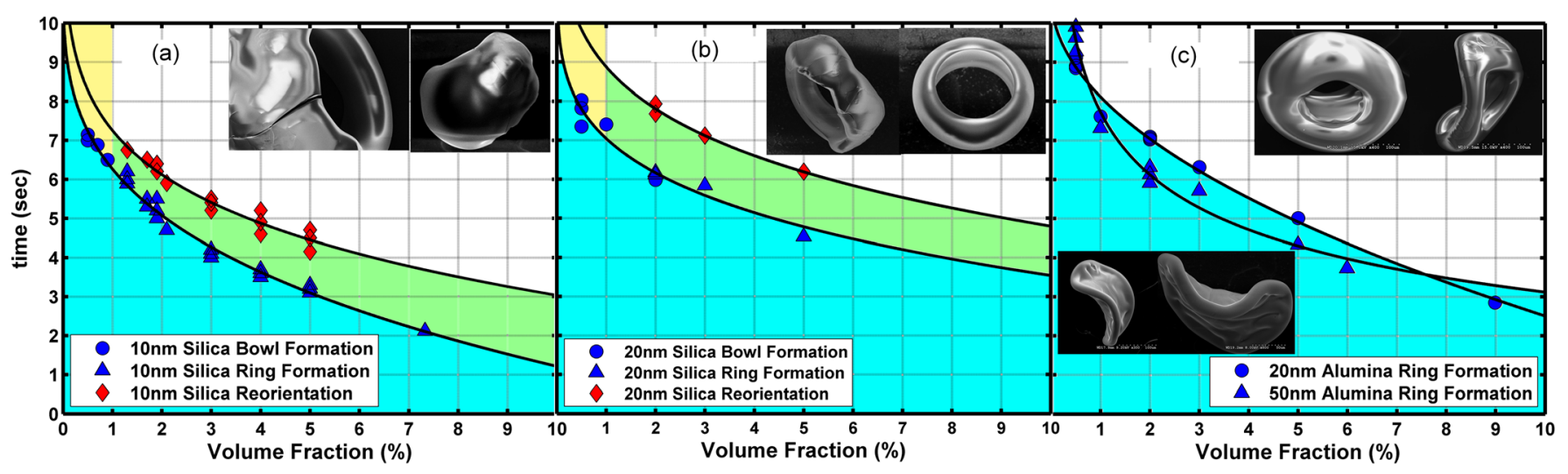

FIG. 5. Phase diagram comparison of silica and alumina droplets. (a) $10 \mathrm{~nm}$ silica: Inset SEM images of ring, (b) $20 \mathrm{~nm}$ silica: Inset SEM images of ring, (c) $20 \mathrm{~nm}$ alumina and $50 \mathrm{~nm}$ alumina: Inset SEM images of disc and rings.

observed in the case of $20 \mathrm{~nm}$ alumina droplets (Figure 3). These suspensions have high viscosity (14 cP) compared to $50 \mathrm{~nm}$ alumina $(1.17 \mathrm{cP})$ and low surface tension $(50 \mathrm{mN} / \mathrm{m}$ compared to $57 \mathrm{mN} / \mathrm{m}$ ), yielding structures that differ from bowls and rings. The structures formed from these droplets have a disk-like geometry surrounded by a toroidal edge, which differs from the hollow structure geometries observed in other nanofluids. The $20 \mathrm{~nm}$ alumina droplets yield structures of higher aspect ratio compared to the structures formed from $20 \mathrm{~nm}$ silica droplets (higher surface tension) as seen in Figure 3. This is due to the reduced recirculation in the high viscosity droplet and acute shape deformation due to low surface tension. Low recirculation implies lower particle migration rate. Low surface tension yields structures of higher aspect ratios compared to the high surface tension droplets. This combined effect results in a flat disc shaped geometry with an annulus at the edge of the structure due to centrifugal force induced from droplet rotation along the longitudinal levitation axis caused by secondary acoustic streaming $^{10,11,15,16}$ (Figure 2 inset).

The structure formation phases, evaporation, agglomeration, and structure reorientation are illustrated using phase diagrams for different fluids (Figure 5). The regions in the time vs. particle concentration domain, separated by solid lines, represent the distinct phases of the structure formation process. The blue region corresponds to the liquid phase evaporation phase, recirculation, particle migration, and early agglomeration. Recirculation decreases with viscosity, which decreases the evaporation rate. At a constant heat flux, the droplet heat absorption rate is proportional to the area exposed to heating. Structure formation is slower when viscosity increases and/or when surface tension decreases. The evaporation phase is longer for $20 \mathrm{~nm}$ silica compared to $10 \mathrm{~nm}$ silica (Figures 5(a) and 5(b)). Even though viscosity of the $20 \mathrm{~nm}$ silica suspension is lower, especially at concentrations higher than $3 \%$ vol., the effect on evaporation rate caused by lower surface tension is dominant, thereby increasing $t_{f}$. The $20 \mathrm{~nm}$ alumina cases in Figure 5(c) present the extreme effects of high viscosity and low surface tension, as the liquid evaporation rate reduces and the structure formation is slightly slower compared to high viscosity $50 \mathrm{~nm}$ alumina. The insets in Figures 5(a)-5(c) shows the SEM images of the structures typically observed at different regimes of the phase plot.
Structure reorientation was observed in the cases of $10 \mathrm{~nm}$ and $20 \mathrm{~nm}$ silica of ring structures formed at particle concentrations higher than $\varphi=1 \%$ vol. caused by geometric asymmetries due to non-uniform particle agglomeration ${ }^{6,10,15}$ (Figures 5(a) and 5(b)). The area between the structure reorientation and structure formation lines represents the region at which the interior of the structures is at the final stage of agglomeration as the internal geometry still changes, and these mass shifts within the structure ultimately cause structure reorientation. However, such reorientation was not seen for the $20 \mathrm{~nm}$ and $50 \mathrm{~nm}$ alumina droplets due to the lower recirculation caused by high viscosity. Relatively slower recirculation and slower structure formation allow the particles to agglomerate in a more uniform manner forming well defined symmetric structures (Figure 2 and 5 insets).

In summary, the structure formation from nanoparticle suspensions in a droplet is a function of the relative magnitudes of the agglomeration, evaporation, and deformation time scales. The final diameter ratio correlates well with the deformation to agglomeration time scale ratio for all droplet nanosuspensions regardless of type, size, and composition. Bowls form consistently at high $\mathrm{t}_{\mathrm{g}} / \mathrm{t}_{\mathrm{e}}$ and of rings at low $\mathrm{t}_{\mathrm{g}} / \mathrm{t}_{\mathrm{e}}$. The viscosity increase also affected the final structure morphology such as ring formation at low $10 \mathrm{~nm}$ silica concentrations at which normally bowls form. Surfactant was used to decrease surface tension with the overall effect of decreasing $t_{\mathrm{g}} / \mathrm{t}_{\mathrm{e}}$ thus increasing the final size of the final structure. Low surface tension also favors the formation of rings due to the increase in droplet aspect ratio. The phase diagrams presented in this work are a different form of representing the length of these phases as highlighted regions and thresholds indicating the transition from one phase to the next.

${ }^{1}$ H. K. Chan, Colloids Surf., A 284, 50 (2006).

${ }^{2}$ F. Iskandar, A. B. D. Nandiyanto, K. M. Yun, C. J. Hogan, K. Okuyama, and P. Biswas, Adv. Mater. 19.10, 1408 (2007).

${ }^{3}$ P. J. Yunker, T. Still, M. A. Lohr, and A. G. Yodh, Nature 476, 308 (2011).

${ }^{4}$ I. I. Smalyukh, O. V. Zribi, J. C. Butler, O. D. Lavrentovich, and G. C. L. Wong, Phys. Rev. Lett. 96, 177801 (2006).

${ }^{5}$ W. J. Xie, C. D. Cao, Y. J. Lu, Z. Y. Hong, and B. Wei, Appl. Phys. Lett. 89, 214102 (2006).

${ }^{6}$ W. J. Xie and B. Wei, Appl. Phys. Lett. 90, 204104 (2007).

${ }^{7}$ E. Tijerino, S. Basu, and R. Kumar, J. Appl. Phys. 113, 034307 (2013).

${ }^{8}$ D. Milanova and R. Kumar, Appl. Phys. Lett. 87, 233107 (2005). 
${ }^{9}$ R. Kumar, E. Tijerino, A. Saha, and S. Basu, Appl. Phys. Lett. 97, 123106 (2010).

${ }^{10}$ A. Saha, S. Basu, and R. Kumar, Appl. Phys. Lett. 100, 204104 (2012).

${ }^{11}$ A. Saha, S. Basu, and R. Kumar, J. Fluid Mech. 692, 207-219 (2012).

${ }^{12} \mathrm{~W}$. A. Sirignano, Fluid Dynamics and Transport of Droplets and Sprays, 2nd ed. (Cambridge University Press, New York, 2010).
${ }^{13}$ R. Vehring, Pharm. Res. 25(5), 999 (2008).

${ }^{14}$ L. G. B. Bremer, P. Walstra, and T. van Vliet, "Estimations of the aggregation time of various colloidal systems," Colloids Surf., A 99, 121 (1995).

${ }^{15}$ E. G. Lierke, Acta Acust. Acust. 88, 206-217 (2002).

${ }^{16}$ A. L. Yarin, G. Brenn, O. Kaster, D. Rensik, and C. Tropea, J. Fluid Mech. 399, 151 (1999). 